De Leo, D. (2002) Why are we not getting any closer to preventing suicide? British Journal of Psychiatry, $\mathbf{~} \mathbf{8 I}$, 372-374

De Leo, D., Klieve, H. \& Milner, A.(2006) Suicide in Queensland, 2002-2004. Mortality Rates and Related Data. Australian Institute for Suicide Research and Prevention.

Kapusta, N. D., Etzersdorfer, E., Krall, C., et al (2007) Firearm legislation reform in the European Union: impact on firearm availability, firearm suicide and homicide rates in Austria. British Journal of Psychiatry, 191, 253-257.

D. De Leo Australian Institute of Suicide Research and Prevention, Griffith University, Queensland, Australia. Email:

diegodeleol @gmail.com

H. Klieve, M. Barnes Australian Institute of Suicide Research and Prevention, Griffith University, Queensland, Australia

doi: 10.1192/bjp.191.6.562a

Authors' reply: McPhedran \& Baker point out an unsolved problem of Australian suicide research. There are concerns about the quality of mortality data and statistics based upon them. Therefore, they urge researchers to approach Australian firearms data with caution. The authors cite a letter to the Medical Journal of Australia (De Leo, 2007) which highlighted inconsistencies in Australian mortality data since the year 2001 and called for standardised certification procedures of deaths according to ICD-10 and for other improvements of death registries. However, in Austria autopsies are performed when there is any uncertainty regarding the cause of death. The autopsy rate is high, with a mean rate of $29 \%$ in 1991-2000 (Waldhoer et al, 2003). If the cause of death is not clear, an additional investigation by Statistics Austria takes place. Statistics Austria registers deaths as suicide if that is the most probable cause of death. The International Classification of Diseases (ICD-8, -9, -10) has been applied for many years and there are no signs of a decrease in the data quality of Statistics Austria. The work reported by Kapusta et al (2007) is based on these data.

Furthermore, De Leo (2007) realistically states that some underreporting is ubiquitous and has to be tolerated in suicide statistics. On the other hand, underreporting of firearm deaths seems less probable than underreporting of, for example, deaths due to poisonings (with longer survival periods), which tend to be classified as disease-related deaths.
We agree with McPhedran \& Baker that Australian firearm laws should be reevaluated on the basis of more reliable data, but as long as sufficient evidence is not available, theoretical assumptions that Australian firearm laws had no life-saving effects remain speculative. This applies also to Europe where independent scientific evaluations of firearm law are still rare.

De Leo, D. (2007) Suicide mortality data needs revision. Medical Journal of Australia, 186, 157.

Waldhoer, T., Berzlanovich, A., Vutuc, C., et al (2003) Rates of postmortem examination in Austria: the effect of distance between location of death and site of examination. Journal of Clinical Epidemiology, 56, $891-895$

N. D. Kapusta Department of Psychoanalysis and Psychotherapy, Medical University of Vienna, Austria. Email: nestor.kapusta@meduniwien.ac.at

E. Etzersdorfer Furtbach Hospital for Psychiatry and Psychotherapy, Stuttgart, Germany

G. Sonneck Institute for Medical Psychology, Medical University of Vienna, Austria

doi: 10.1192/bjp.191.6.563

\section{Cardiovascular risk with antipsychotics: case-control study or survey?}

Mackin et al (2007) highlighted the significantly higher risk of cardiovascular and metabolic diseases in people with severe mental illness. Rates of metabolic syndrome and cardiovascular risk similar to those in schizophrenia have been reported in bipolar disorders, and atypical antipsychotics have been approved for the treatment of the latter (Fagiolini et al, 2005; Birkenaes et al, 2007). This implies that all such populations should be studied for putative longterm adverse outcomes, as in the timely study of Mackin et al (2007).

However, some methodological issues need clarification. Mackin et al state that their study is a case-control study, but by definition a case-control study starts with an outcome and investigates exposure to putative risk factors in groups with and without the outcome (Lewallen \& Courtright, 1998), generating a measure of relative risk with regard to a given risk factor. Mackin et al started with a group with mental illness on antipsychotics and studied the prevalence of metabolic disease and cardiovascular risk compared with controls. Thus the study has really used a survey design with a control group. The use of a control group alone does not justify the label of a 'case-control study'.

As an important corollary of this distinction, the sample size is rather low for a community-based survey. Mackin et al mention that comparative data for physical comorbidity in people with diagnoses other than schizophrenia are sparse; unfortunately, the study fails to generate such data owing to the inadequate sample size. We feel that Mackin et al have gone beyond their brief to analyse the effect of individual factors such as diagnoses, type of antipsychotic and smoking; not surprisingly, they failed to emerge with convincing findings as the sample was underpowered to generate such data.

Finally, we wonder whether the inclusion of several patients with depression and anxiety is appropriate for a study on 'severe mental illness', a term traditionally reserved for psychotic and bipolar disorders. The common denominator seems to be 'treated with antipsychotics' rather than 'severe mental illness'. It is interesting to note that the type of antipsychotics had no impact on the outcome measures (except serum insulin). If replicated in a much larger community sample, this so-called negative finding could have far-reaching implications regarding choice of treatment. Another important factor in the secondary analysis could have been the duration of treatment with antipsychotics and the dosages used. In a recent study, higher doses of medication were associated with increased cardiovascular risk scores (Osborn et al, 2006). Including the dosage and duration of antipsychotics in the analysis could provide important insights regarding the true impact of antipsychotics on the outcome measures.

This study, like several others, reiterates that patients treated with antipsychotics are at heightened risk for cardiovascular events and metabolic syndrome. Longitudinal studies are needed to explore the relative contribution of putative aetiological factors to physical comorbidity in severe mental illness.

Birkenaes, A. B., Opjordsmoen, S., Brunborg, C., et al (2007) The level of cardiovascular risk factors in bipolar disorder equals that of schizophrenia: a comparative study. Journal of Clinical Psychiatry, 68, 917-923.

Fagiolini, A., Frank, E., Scott, J. A., et al (2005)

Metabolic syndrome in bipolar disorder: findings from the Bipolar Disorder Center for Pennsylvanians. Bipolar Disorders, 7, 424-430.

Lewallen, S. \& Courtright, P. (1998) Epidemiology in practice: case-control studies. Community Eye Health, II, 57-58 
Mackin, P., Bishop, D., Watkinson, H., et al (2007) Metabolic disease and cardiovascular risk in people treated with antipsychotics in the community. British Journal of Psychiatry, 191, 23-29.

Osborn, D. P. J., Nazareth, I. \& King, M. B. (2006) Risk for coronary heart disease in people with severe mental illness: cross-sectional comparative study in primary care. British Journal of Psychiatry, 188, 27I-277.

A. Banerjee Postgraduate Institute of Medical Education and Research, Chandigarh, India. Email: dr.banerjee@gmail.com

D. Basu Postgraduate Institute of Medical Education and Research, Chandigarh, India doi: 10.I192/bjp.191.6.563a

Authors' reply Banerjee \& Basu are correct to point out that our study does not have a case-control design in the purest epidemiological sense, that is a study in which patients who have developed a disease are identified and their past exposure to aetiological factors is compared with that of controls. Our study is conceptually similar to the case-control design, although we accept that both our study and case-control studies are inherently vulnerable to methodological weaknesses as discussed recently (Lee et al, 2007). We selected individuals who had had a diagnosis of severe mental illness and antipsychotic treatment to ascertain whether this population was at increased risk for cardio-vascular and metabolic disease compared with a control group.

We also accept, and acknowledge in our paper, that the sample size is relatively small. However, we found highly statistically significant differences between patients and controls across a number of outcomes. The analysis of effect of diagnosis, type of antipsychotic medication and smoking status was not a primary aim but was a secondary analysis. Increasing the sample size further might have added power to the study to detect differences in these variables. Notwithstanding, emerging evidence from studies in people with bipolar disorders points to an excess of cardiovascular and metabolic disease comparable to that in schizophrenia, suggesting that the similar rates across our diagnostic groups is a true finding.

Banerjee \& Basu question the appropriateness of the term 'severe mental illness' The vast majority $(75.6 \%)$ of patients had a diagnosis of schizophrenia, bipolar disorder or schizoaffective disorder. Many in the 'other' category (comprising 24.4\%) had experienced psychotic depression and other severe depressive disorders requiring antipsychotic treatment. Although the use of the word 'severe' may be questioned, we are confident that this is appropriate.

We await larger, prospective studies designed specifically to tease out the aetiological factors that contribute to an excess of cardiovascular and metabolic risk and ultimately an excess mortality rate in this population. We hope our study has gone some way to highlighting the need for vigilant monitoring and appropriate intervention in this high-risk group.

Lee, W., Bindman, J., Ford, T., et al (2007) Bias in psychiatric case-control studies: literature survey. British Journal of Psychiatry, 190, 204-209.

P. Mackin Institute of Neuroscience,

Newcastle University, Newcastle, UK.

Email: paul.mackin@ncl.ac.uk

P. Gallagher Institute of Neuroscience,

Newcastle University, Newcastle, UK

doi: 10.II92/bjp.191.6.564

\section{Psychiatry and faith-based organisations}

Leavey \& King (2007) provide a usefu overview of the relationship between the clergy and psychiatry. Although I wholeheartedly agree that there should be partnerships between psychiatry and religious sectors, I found the discussion to be onesided. Leavey \& King say little about what religion can offer psychiatry. It is well recognised that religious states are often misdiagnosed as mental illness (Dein, 2004). Religious professionals can play a pivotal role in teaching mental health professionals about normative religious experiences and thus enable them to make better informed diagnoses. It is not just that religious professionals need to be educated about mental illness but it is also vital that psychiatrists understand religious experience.

Of course ideas about mental illness reflecting sin still exist in some religious communities, but in my own fieldwork among Orthodox Jews and Pentecostal Christians it is evident that rabbis and pastors increasingly recognise mental illness as a state independent of moral indiscretion. Even in communities where extreme religious experiences, such as hearing God's voice, are prevalent, religious leaders are able to differentiate these experiences from the symptoms of severe mental disorder (Dein
\& Littlewood, 2007). Similarly they are able to differentiate psychoses from states of spirit possession (which themselves require stringent criteria for their diagnosis within the religious context).

Beyond this, there is emerging evidence that religion can play an important role in facilitating coping with life stressors (Pargament, 1997). Mental health professionals need to be knowledgeable about the circumstances in which referrals to religious professionals are appropriate. They should be aware that for religious believers, prayer and ritual may play a central role in the healing process. Of course involvement in such activities may influence pathways to care but there is ample evidence that religious and biomedical forms of healing can work well together: biomedicine healing the body and religion healing the soul (Littlewood \& Dein, 1995).

Finally I take issue with the statement that biomedical and spiritual models of illness are necessarily conflicting. Spirituality and biomedicine offer different types of explanations for patients' problems. The art of medicine should be to learn how to combine different treatments in order to provide more holistic care to patients.

Dein, S. (2004) Working with patients with religious beliefs. Advances in Psychiatric Treatment, 10, 287-294.

Dein, S. \& Littlewood, R. (2007) The voice of God. Anthropology and Medicine, 14, 213-228.

Leavey, G. \& King, M. (2007) The devil is in the detail: partnerships between psychiatry and faith-based organisations. British Journal of Psychiatry, 191, 97-98.

Littlewood, R. \& Dein, S. (1995) The effectiveness of words: religion and healing among the Lubavitch of Stamford Hill. Culture, Medicine and Psychiatry, I, 339-383.

Pargament, K. (1997) The Psychology of Religion and Coping: Theory, Research, Practice. Guilford Press.

S. Dein Centre for Behavioural and Socia Sciences in Medicine, University College London, Charles Bell House, 67 Riding House Street, London WIN 8AA, UK. Email: s.dein@ucl.ac.uk

doi: 10.II92/bjp.19I.6.564a

Faith and other religious terms are still not considered an explicit language of psychiatry. Faith is a multilayered phenomenon, involving a belief about things of which we are uncertain accompanied by an expectancy and/or conviction (Clarke, 2003). The Bible defines faith as being sure of what people hope for and certain of what they do not see (Hebrews 11:1). Faith is considered a nebulous concept and its 